\title{
Study on the adsorption of poly(o-ethoxyaniline) nanostructured films using atomic force microscopy
}

\author{
F.L. Leite ${ }^{\mathrm{a}, \mathrm{b}}$, L.G. Paterno ${ }^{\mathrm{b}}{ }^{\text {, C.E. Borato }}{ }^{\mathrm{a}, \mathrm{b}}$, P.S.P. Herrmann ${ }^{\mathrm{b}}$, \\ O.N. Oliveira $\mathrm{Jr}^{\mathrm{a}}$, L.H.C. Mattoso ${ }^{\mathrm{b}, *}$ \\ a Instituto de Física de São Carlos, USP, CP 369, 13560-970, São Carlos, SP, Brazil \\ ${ }^{\mathrm{b}}$ Embrapa Instrumentação Agropecuária, CP 741, 13560-970, São Carlos, SP, Brazil
}

Received 17 March 2005; received in revised form 24 June 2005; accepted 7 July 2005

\begin{abstract}
Nanostructured films of poly(o-ethoxyaniline) (POEA) were studied by atomic force microscopy (AFM), which indicated a globular morphology for films containing one or more layers of POEA. Consistent with the nucleation and growth model for the adsorption process, the mean roughness and fractal dimension were found to increase with the time of adsorption and with the number of POEA layers in the initial stages of adsorption, reached maximum values and then decreased after $10 \mathrm{~min}$ of adsorption or after deposition of five POEA layers. Such behavior has been explained in terms of the decrease in the film irregularities, with voids being filled with polymeric material leading to smoother surfaces. (C) 2006 Elsevier Ltd. All rights reserved.
\end{abstract}

Keywords: Poly(o-ethoxyaniline); Adsorption; Atomic force microscopy

\section{Introduction}

Nanostructured films of conducting polymers have been extensively investigated as they can be used in a variety of applications, including sensors, optical storage, charge storage and electrochromic devices [1]. These films may be obtained with techniques such as the Langmuir-Blodgett (LB) [2] or the layer-by-layer method (LbL) [3], which offer precise control of thickness and molecular architecture. In the LbL films, polymer adsorption is in most cases driven by ionic interactions, with oppositely charged materials from aqueous solutions being deposited alternately on a solid substrate. Secondary interactions-e.g. hydrogen bonding — may also be important for adsorption [4], as it has been shown for polyanilines, for which H-bonding contributes even when the molecules are protonated [5]. One consequence of the adsorption governed by H-bonding is that multilayers of the same material may be deposited on the same substrate, leading also to the so-called non-self-limiting adsorption [6]. The layer thickness can therefore be controlled by altering the interactions responsible for adsorption, which is carried out

\footnotetext{
* Corresponding author. Tel.: +55163374 2477; fax: +551633725958. E-mail address: mattoso@cnpdia.embrapa.br (L.H.C. Mattoso).
}

0032-3861/\$ - see front matter (C) 2006 Elsevier Ltd. All rights reserved. doi:10.1016/j.polymer.2005.07.108 by changing materials and experimental conditions such as $\mathrm{pH}$, ionic strength and concentration of the solutions. For parent polyaniline and derivatives, for instance, film properties may vary widely due to the interplay of the adsorption mechanisms [5], and therefore a thorough analysis of the capabilities of LbL films requires use of several characterization techniques.

Because LbL are essentially surface films, one method that has proven suitable for characterization is the scanning force microscopy, especially the atomic force microscopy (AFM) [7]. From AFM results, a number of features of LbL films of polyanilines could be captured not only in terms of the film properties but also of the adsorption mechanisms. For protonated (doped) polyanilines, ionic interactions made it possible to assemble LbL films with various water-soluble polyanions, including sodium polystyrene sulfonate [8] and sodium polyvinyl sulfonate [4]. Undoped polyaniline was assembled with the nonionic water soluble polymers poly(vinylpyrrolidone), poly(vinyl alcohol), poly(acrylamide) and poly(ethylene oxide) [4]. In other cases, ionic interactions and $\mathrm{H}$-bonding were found to coexist during polymer adsorption, which depended on the $\mathrm{pH}$ of the solution [5]. At $\mathrm{pH}>5$, for both poly (o-methoxyaniline) (POMA) and poly (o-ethoxyaniline) (POEA), adsorption was attributed entirely to H-bonding since the polymers were not expected to be charged [6]. In fact, through H-bonding POMA and POEA layers could be adsorbed onto already deposited POMA (or POEA), with no 
(a)

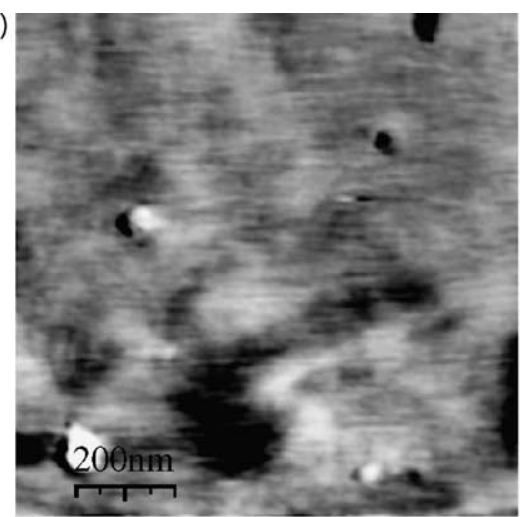

(c)

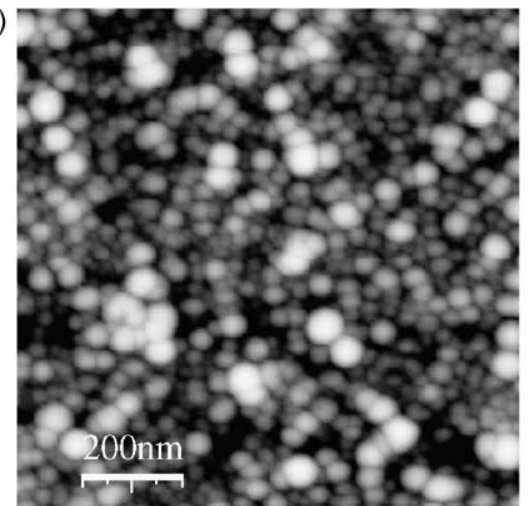

(e)

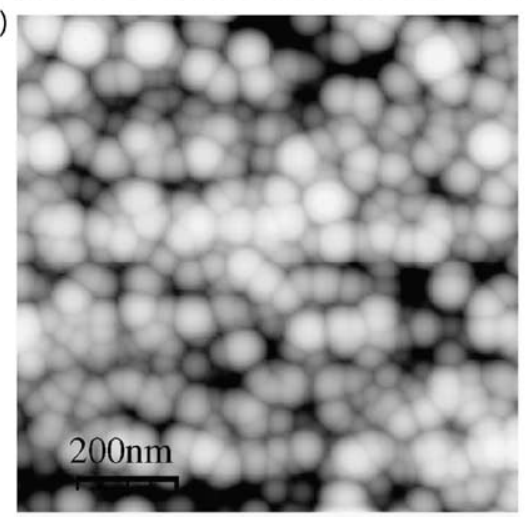

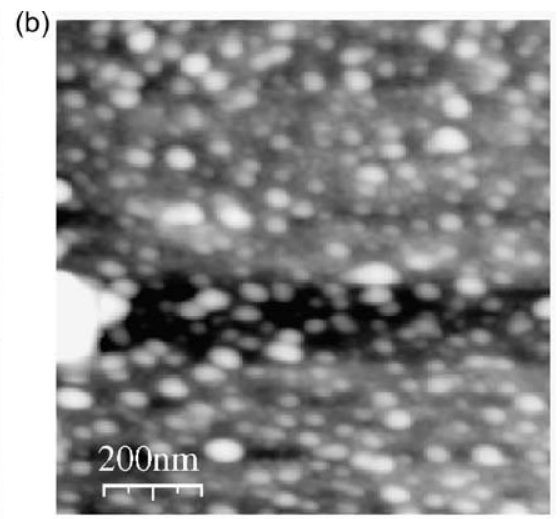

(d)

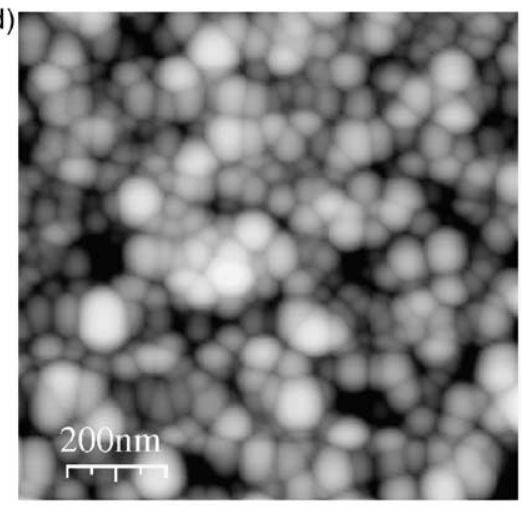

(f)

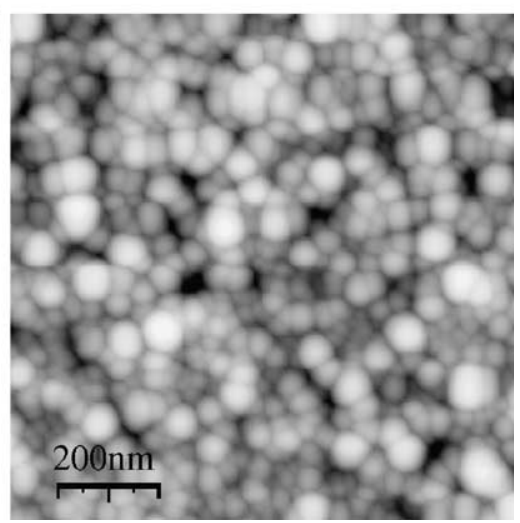

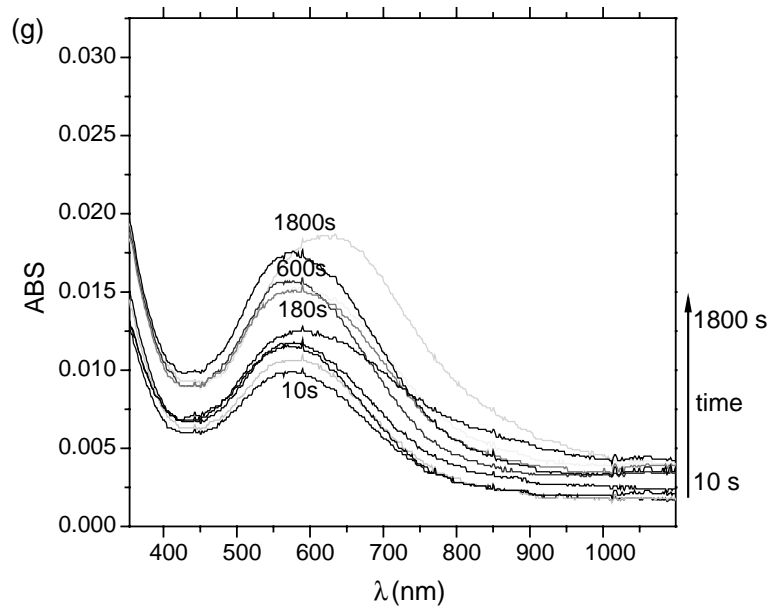

Fig. 1. AFM images of POEA films produced from a pH 3 solution for distinct immersion times. (a) 0.0 min (i.e. bare glass); (b) 0.5 min; (c) $1.0 \mathrm{~min}$; (d) 3.0 min; (e) $10.0 \mathrm{~min}$; (f) $30.0 \mathrm{~min}$. In (g) are shown the spectra with increasing absorption for increasing immersion times, from $10 \mathrm{up}$ to $1800 \mathrm{~s}$. Some of the curves are labeled with the corresponding immersion time. 
intercalating polymer layer, and even reaching the nonselflimiting process where the polymer layer grows indefinitely [6].

The kinetics of adsorption of POMA layers has been found to consist of a two-step process. The first, fast process is due to nucleation while the second is attributed to a growth mechanism described with a Johnson-Mehl-Avrami function [9]. This is consistent with the proposal of Lvov and Decher [10], who stated that the adsorption of polyelectrolytes is based on two distinct stages: in the first, some polymeric segments are anchored to the substrate, while in the second such anchored segments serve as nucleation sites for additional polymer adsorption. Saturation of adsorption is a result of the equilibrium between the number of occupied sites and the electrostatic repulsion between the ionized groups of the polymer chains.

In this paper, we analyze the morphology and adsorption processes for POEA layers using AFM images. Parameters such as roughness, relative surface coverage and fractal dimension are used to interpret the data. The analysis of surface roughness, for instance, enables one to describe theoretically and quantitatively real surfaces, especially at the short-length scales $[11,12]$.

\section{Experimental section}

POEA was chemically synthesized according to the method described in Ref. [13]. The polymer obtained is a dark precipitate, with expected weight average molecular weight $\left(M_{\mathrm{w}}\right)$ of $\sim 41,400 \mathrm{~g} \mathrm{~mol}^{-1}$ and polydispersity $\left(M_{\mathrm{w}} / M_{\mathrm{n}}\right)$ of 2.3 [13]. Polymeric solutions were prepared by dissolution of POEA in a mixture of $\mathrm{HCl}$ and ultra-pure water, 1:49 v/v, under constant stirring for $18 \mathrm{~h}$. The final concentration of polymer was $0.5 \times 10^{-3} \mathrm{~mol} \mathrm{~L}^{-1}$ and the $\mathrm{pH}$ of each solution was adjusted to 3 by adding $0.1 \mathrm{M} \mathrm{HCl}$ solution. Substrates were prepared with glass slides $(10 \times 10 \times 1 \mathrm{~mm})$ previously cleaned in $\mathrm{H}_{2} \mathrm{SO}_{4} / \mathrm{H}_{2} \mathrm{O}_{2}, 7: 3 \mathrm{v} / \mathrm{v}$ solutions for $1 \mathrm{~h}$, followed by extensive washing in ultra-pure water. The slides were then immersed into a $\mathrm{H}_{2} \mathrm{O} / \mathrm{H}_{2} \mathrm{O}_{2} / \mathrm{NH}_{4} \mathrm{OH}$ 5:1:1 v/v solution for $40 \mathrm{~min}$ and again washed with large amounts of ultra pure water. Here, we have studied two types of film. In the first one single layer is adsorbed onto glass slides by immersing the slides into the POEA solution for a given period of time, which was varied to investigate the adsorption kinetics. After adsorption, the film/substrate system was washed in an aqueous solution of same $\mathrm{pH}$ and dried with a gentle nitrogen flow. In the second set of samples, up to 10 POEA layers were adsorbed on the same substrate by repeating the procedure above, with no intercalating layers. Even though POEA was charged, deposition of further POEA layers was made possible because of H-bonding, as discussed above. The growth of the POEA layers was monitored by measuring the UV-vis absorption with a UV-vis spectrophotometer Shimadzu UV-1601 PC.

AFM images were taken in a Topometrix microscope, model Explorer TMX 2010, using silicon nitride tips (V shape) with spring constant of $0.09 \mathrm{~N} / \mathrm{m}$. Roughness and surface fractal dimension were calculated using WS $\times$ M 4.0 software from Nanotec Electronica S.L. (copyright $\left.{ }^{(}\right)$November 2003) and scanning probe image processor (SPIP) version 3.1.0.1 from Image Metrology A/S 2003. All images were obtained in the contact mode at a scan rate of $2 \mathrm{~Hz}$.

\section{Results and discussion}

The LbL technique allows film production within short time intervals, and therefore it is important to evaluate the kinetics of polymer adsorption in order to optimize the time of film fabrication. A few minutes are usually sufficient to form a stable and continuous layer of different kinds of materials [8, 14]. Here, we shall describe the evolution of POEA film morphology using AFM. Fig. 1 displays AFM images of a POEA layer adsorbed at different time intervals at $\mathrm{pH} 3$ on a glass substrate. In general, the morphology of a POEA film is characterized by small globules, ranging from 20 to $100 \mathrm{~nm}$ in diameter. The number of globules increases with the time of immersion and then reaches saturation as the substrate is entirely coated. Since we used a $\mathrm{pH}=3$ for the POEA solution, there should be considerable repulsion as POEA is positively charged. The surface of POEA is expected to reflect both the long-range morphology due to the existence of globules, and the short-range morphology corresponding to the region inside the globules. The last term may be dominant for thicker films (two or more layers) [15]. The increase in the amount of adsorbed POEA with increasing times is indicated in the increased absorption in the UV-vis spectra of Fig. 1(g).

The evolution with time of the number of globules (average and maximum) is depicted in Fig. 2, which basically increases during the first minutes of adsorption and then decreases for longer times. Before $1 \mathrm{~min}$ of adsorption, the nucleation process appears to be complete, being followed by the gradual growth of the globules without creation of new sites. The radial growth of globules occurs as a result of the incorporation of new polymeric chains. Finally, coalescence

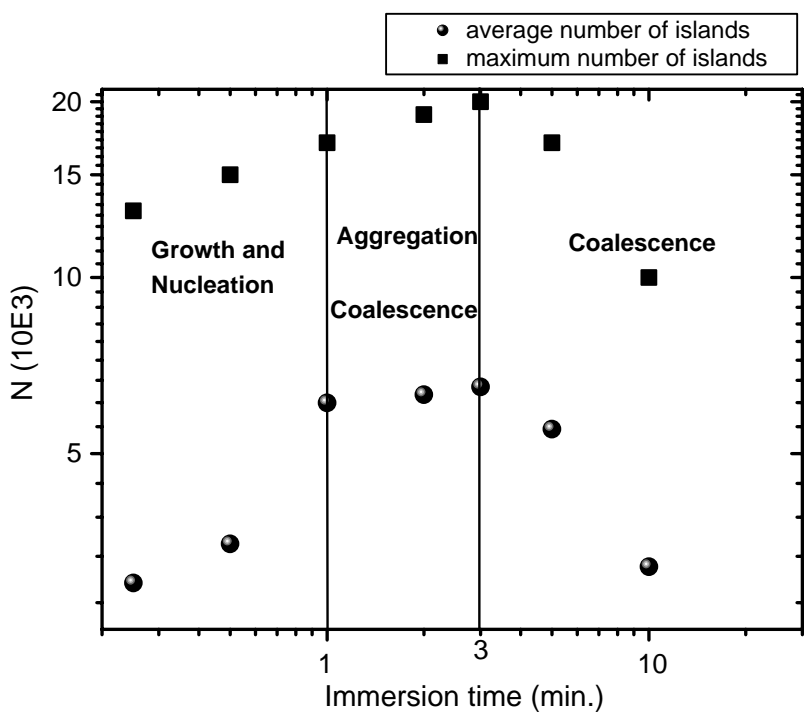

Fig. 2. Average and maximum number $(N)$ of globules vs. time of immersion of the substrate in the POEA solution. Taken from AFM images for $1 \mu^{2}$ area. 


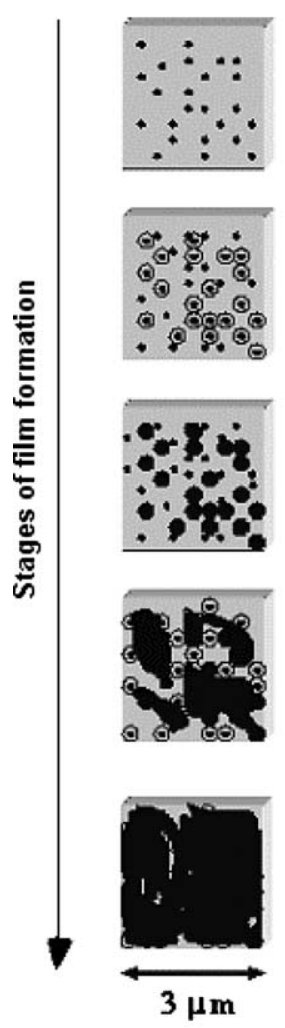

(a)

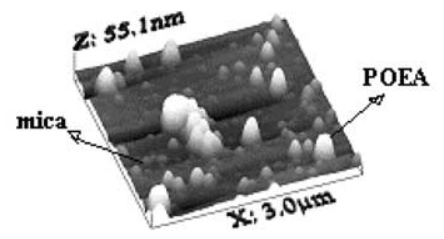

initial stage (nucleation regime)
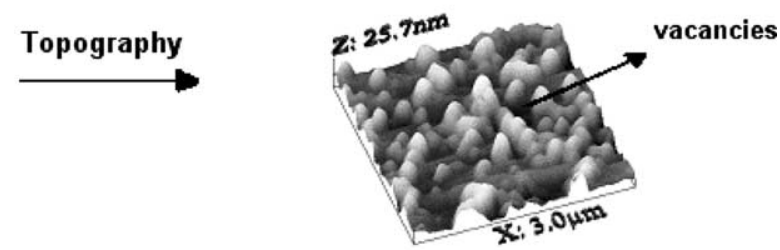

intermediate stage (aggregation regime)

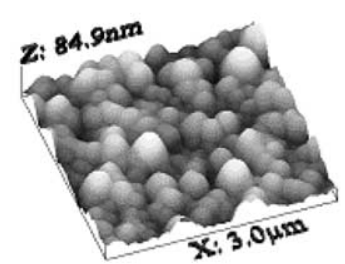

final stage (coalescence regime)

(b)

Fig. 3. (a) Typical model of progressive nucleation, growth and coalescence; (b) AFM images of POEA on muscovite mica for initial, intermediate and final stages of adsorption.

of globules leads to a decrease in their number, these results are according with those presented in the literature for monolayers of octadecylphosphonic acid on mica [16]. The size of the globules increases with time from $20 \mathrm{~nm}$ in the initial stages up to $100 \mathrm{~nm}$ at $3 \mathrm{~min}$ of adsorption. It then tends to decrease after $10 \mathrm{~min}$. These conclusions were based on images obtained from three distinct spots of the same sample. Note that these measurements were taken for POEA films in air. The morphology of the film as it is deposited, in contact with the POEA solution, is different because the domains tend to be larger, probably due to swelling of the polymer chains [17].

In Fig. 3(a) we depict a possible model for thin film growth, which includes nucleation, aggregation and coalescence processes - with the corresponding AFM images for a POEA film in Fig. 3(b). The rationale is based on a computer simulation model according to which polymer molecules adsorb from the bulk at a rate $R$ and then suffers a 2D random walk on the surface with a surface diffusion constant $D$ [18]. Collisions between these (2D) diffusing molecules result in the formation of clusters or globules, which then grow due to the accumulation of additional material [19]. With diffusion, the nucleus not only grows from its initial position but also triggers the formation of other, smaller nuclei, with the morphology becoming flatter. The process of growth and coalescence depends largely on the diffusion rate. If $D$ is large, molecules adsorbed on the surface will probably collide with existing globules. If $R$ is large, there will also be the formation of large globules. Consistent with this explanation is the observation that the growing globules are compact in shape instead of fractal and this implies that desorption from globules and rearrangement into more favorable configuration occurs after than 10 min of adsorption.

The images shown in Fig. 3(b) bring only some snapshots of the growth process. In the upper image, corresponding to the initial stage of growth, a number of scattered domains are seen, which is consistent with a nucleation regime. Considering other images (not shown), we know that this regime lasts up to ca. $60 \mathrm{~s}$. For immersion times higher than $60 \mathrm{~s}$ and up to ca. $180 \mathrm{~s}$, aggregation is observed, as can be seen in the image in the middle of Fig. 3(b), where the substrate is practically fully covered. Coalescence occurs for longer periods of immersion, which is denoted by a smaller number of larger domains.

Another important issue in the POEA film formation is the change in roughness with the time of adsorption. The simplest way to characterize the surface roughness is to determine the roughness parameters RMS (root-mean-square average) and $R_{\mathrm{a}}$ (arithmetic average). Both are functions of the height deviation 


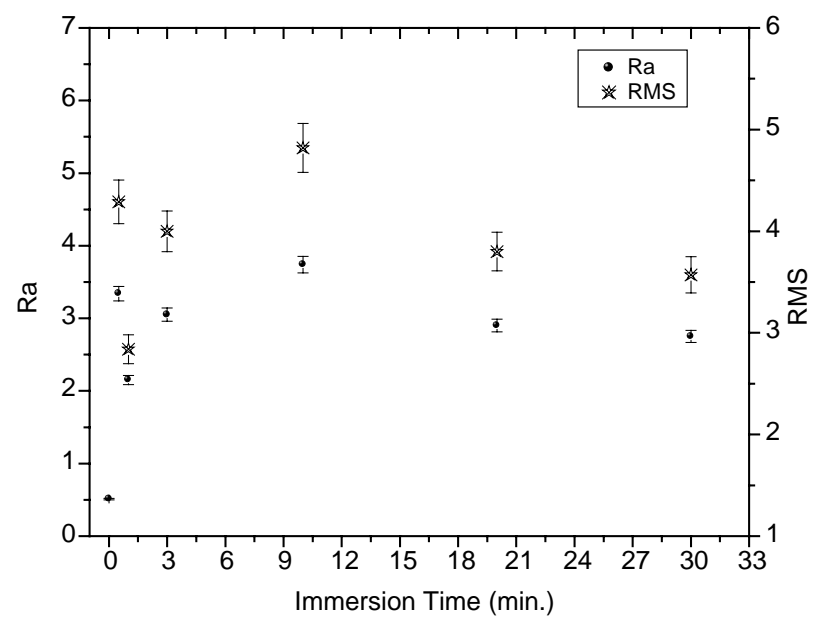

Fig. 4. Roughness vs. immersion time for $1 \mu \mathrm{m}^{2}$ area.

from the mean surface level, as follows:

$\mathrm{RMS}=\left[\frac{1}{N} \sum_{i=1}^{N} Z_{i}^{2}\right]^{1 / 2}$

and

$R_{\mathrm{a}}=\frac{1}{N} \sum_{i=1}^{N}\left|Z_{i}\right|$

where $N$ is the number of data points and $Z_{i}$ is the distance from the mean surface level.

The mean surface level is defined as a reference, being parallel to the general direction of the profile within the limits of the sampling length, such that the sum of the areas contained between this line and those parts of the profile that lie on either side are equal [20]. Fig. 4 shows that the roughness tends to increase with the time of adsorption and then reaches a maximum at $10 \mathrm{~min}$. For longer periods, there is a small decrease and the roughness stabilizes after $20 \mathrm{~min}$. In the first minutes of adsorption, the glass surface is covered with few globules and consequently, the roughness, which is basically the ratio between the height of 'peaks' and 'valleys' on the sample surface, is rather large. As the number of globules increases the roughness becomes a result of the presence of POEA film exclusively, and therefore, it tends to reach a constant value, higher than the bare glass. The small decrease in roughness with the time of adsorption can be related to rearrangements of polymeric chains and globules on the film surface [21]. At longer times, the polymeric chains are able to assume more favorable conformations, which usually tend to be flatter in consequence of the surface energy minimization. The same has been observed in other polyelectrolyte systems, where the surface morphology becomes more compact as the time of adsorption increases [16].

For a globular morphology as observed for the POEA films the concept of fractals is useful for the description of the irregularities on the film surface. Fractal geometry is a branch of mathematics that describes disordered objects using fractional dimensions. The dimension of Euclidian geometry (a)

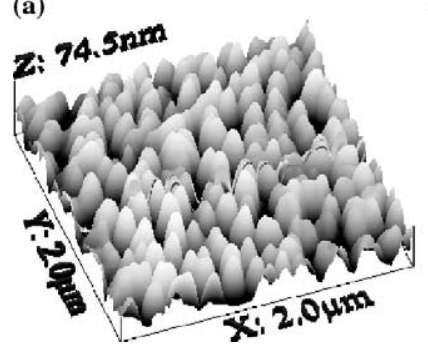

(b)

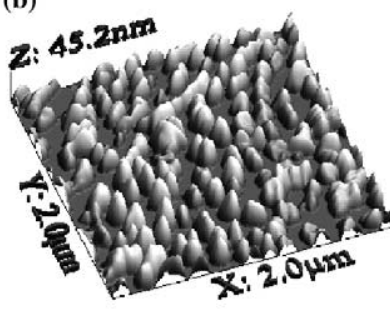

Fig. 5. AFM images of POEA $(2 \times 2 \mu \mathrm{m})$ showing (a) topography and (b) topography with the empty spaces assumed to be filled.

are given as the integers $0,1,2$, and 3 corresponding to dots, lines, planes, and 3D bodies, respectively [22]. This classification, however, is inadequate for the irregular shapes of numerous natural and artificial geometrical objects. One can intuitively assign intermediate dimensional values to such objects. A fractal is a measure of how 'complicated' a selfsimilar figure values to such objects and determine the relative amounts of the surface irregularities for different distance scales [23]. In a rough sense, it measures 'how many points' lie in a given set and is characterized by a scaling law such as $L_{\lambda} \sim \lambda^{1-f}$ is the value of the curve measured with the unit $\lambda$ and $f$ is the fractal dimension. The fractal dimension can be experimentally evaluated through the Minkowski dimension. In this work, though, $f$ was estimated using a different approach, taking into account that an intersection of a plane with a self-similar or a self-affine fractal surface generates selfsimilar lakes (with less materials). This approach is depicted in Fig. 5, where the voids in the image of a POEA film are 'filled' until a certain level. The perimeter $L$ and the area $A$ of the lakes formed were estimated with the $\mathrm{WS} \times \mathrm{M} 4.0$ software and using the relation $L=\alpha f^{\prime} A^{f^{\prime} / 2}$, where $\alpha$ is a constant and $f^{\prime}=f-$ 1 is the fractal dimension of the void perimeter. In order to avoid errors in the fractal dimension measurements, several height thresholds were analyzed, varying $\pm 3 \mathrm{~nm}$, from which the $f^{\prime}$ values were obtained independently of the surface depth. We have used for 'filling' of the void volumes a height value corresponding to $50 \%$ of the maximum height for each image.

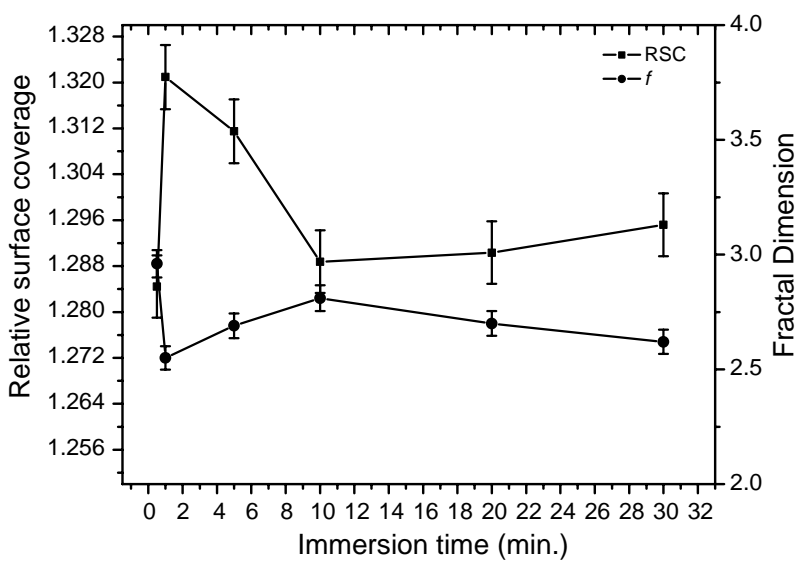

Fig. 6. Fractal dimension $(f)$ and relative surface coverage (RSC) for POEA on glass vs. immersion time. 


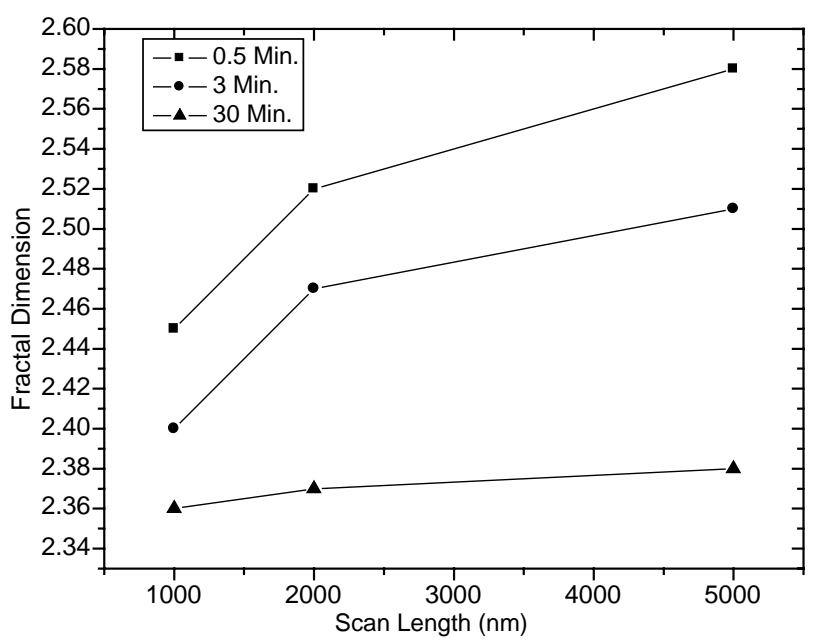

Fig. 7. Fractal dimension for distinct immersion times vs. scan length (one layer).

The variation of this level does not significantly affect the final results.

Fig. 6 displays how $f$ and relative surface coverage (RSC) for POEA films varies with the immersion time. The RSC which is represented by the ratio between the RMS and $R_{\mathrm{a}}$, is also presented. If the height ratio is $x$ and the degree of coverage with smaller globules is $\theta$, then we can express the $\mathrm{RMS} / R_{\mathrm{a}}$ ratio as $[15]$ :

$\frac{\mathrm{RMS}}{R_{\mathrm{a}}}=\frac{\left[x^{2}(1-\theta)+\theta\right]^{1 / 2}}{x(1-\theta)+\theta}$
The fractal dimension $f$ remains between 2 and 3 . Since $f=2$ for a Euclidian surface, an increase above 2 means an increase in film roughness. The highest value for $f$ is observed at $10 \mathrm{~min}$ of adsorption. The coverage factor is stabilized after $10 \mathrm{~min}$ of adsorption which means that the surface is covered with a continuous film right after equilibrium between adsorption and desorption. With the increase in immersion time up to $30 \mathrm{~min}$, the fractal dimension is approximately 2.6. Generally, if a surface is sufficiently flat, both RMS and RA roughness are similar. However, if a surface is rather rough containing a considerable number of islands and voids, the value of RMS is larger than RA. Indeed, for 1 min of adsorption a greater value of $f$ is observed, i.e. there are more irregularities at this stage. In fact, few sites on the glass surface are occupied with POEA globules at this time; as the adsorption continues, more sites are occupied and the coverage of the surface with POEA film increases.

For immersion times up to $10 \mathrm{~min}$ a slight decrease of fractal dimension is accompanied by a considerable change (increase) in the RSC due to the characteristic flatness of surface and more polymers chains adsorbed after $10 \mathrm{~min}$. The surfaces of these irregularities are not taken into account in the calculation of 'real' surface areas. To a certain extent this is confirmed by comparison of fractal dimension values for the same film obtained for different scan lengths (Fig. 7). The results show that the fractal dimension of the sample increases with the scan length and smaller immersion times. For long immersion times, e.g. $30 \mathrm{~min}$, the film exhibits a self-similar behavior with almost no change in $f$ with the scan length being varied by $1-2$ orders of magnitude. However, for shorter immersion times,

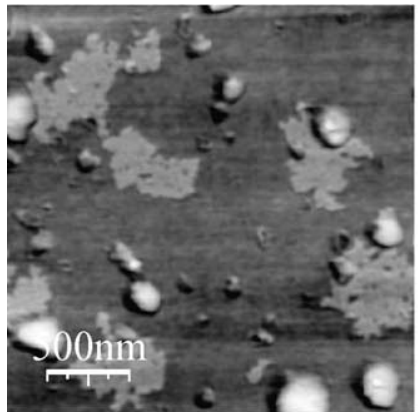

(a)

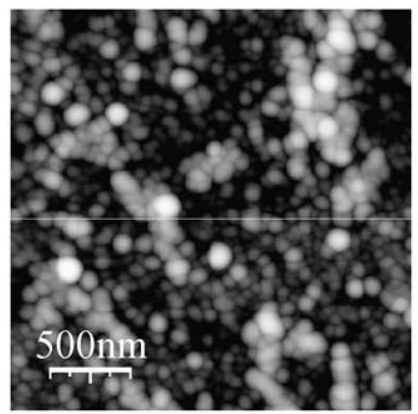

(d)

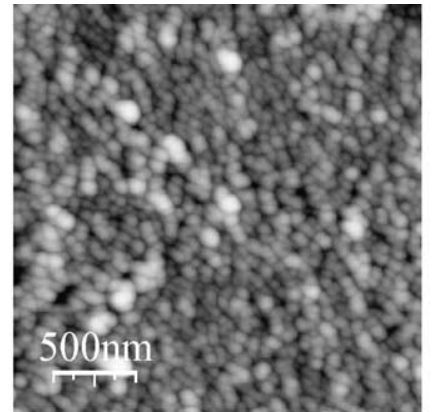

(b)

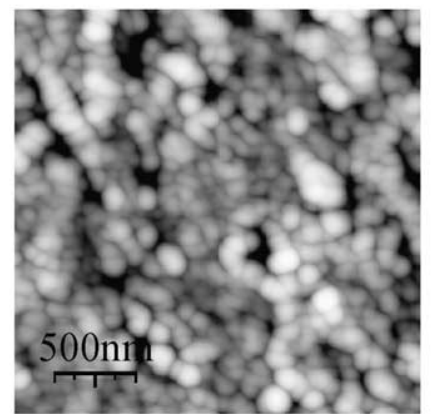

(e)

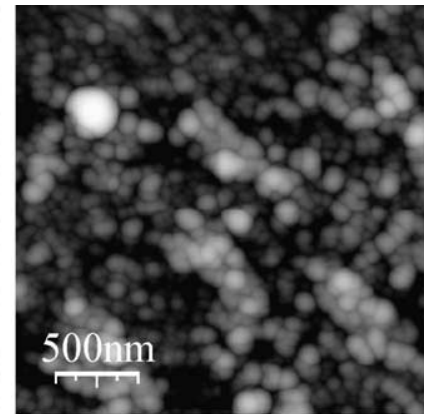

(c)

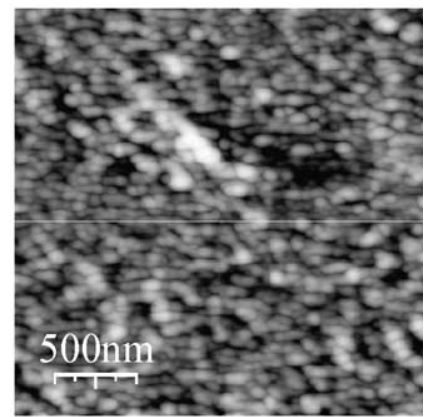

(f)

Fig. 8. AFM images of POEA in $\mathrm{pH}=3$ for distinct number of layers. (a) Glass surface; (b) one layer; (c) three layers; (d) five layers; (e) seven layers and (f) nine layers. 


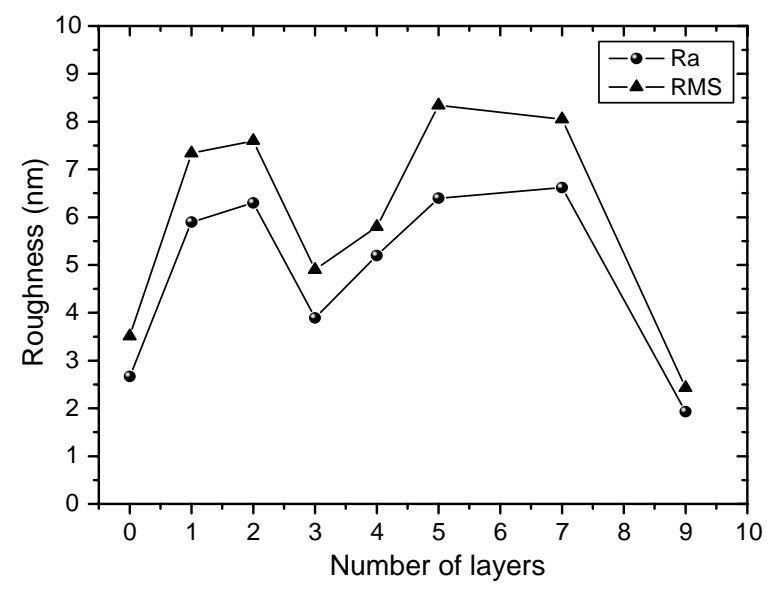

Fig. 9. Roughness vs. number of layers for POEA.

Fig. 7 indicates that distinct morphologies are observed at different length scales.

We have also investigated the morphology of films containing more layers of POEA, with the multilayer being produced by successive immersion of a glass slide into the POEA solution during $3 \mathrm{~min}$. After each immersion, the deposited film was washed in aqueous solution of the same $\mathrm{pH}$ and dried with nitrogen flow. Fig. 8 presents AFM images for films containing different numbers of POEA layers, indicating a globular morphology in all cases. This result is in agreement with the model proposed for POEA adsorption, where small nuclei are first adsorbed and then serve as nucleation sites for further polymer deposition. For multilayer films of POEA alternated with sulfonated lignin (SL) layers, however, the globular morphology is absent when SL is adsorbed on top of POEA layers. In fact, the surface roughness for LbL films of POEA and SL depends on the topmost layer, since the films are much rougher when POEA is the top layer in comparison to those with SL in the top layer [24].

With a globular morphology, regardless of the number of POEA layers, one could expect a continuous increase in surface roughness. However, this expectation is not fulfilled, as indicated in Fig. 9. The roughness is related to the presence of islands and voids on the substrate surface. For the first POEA

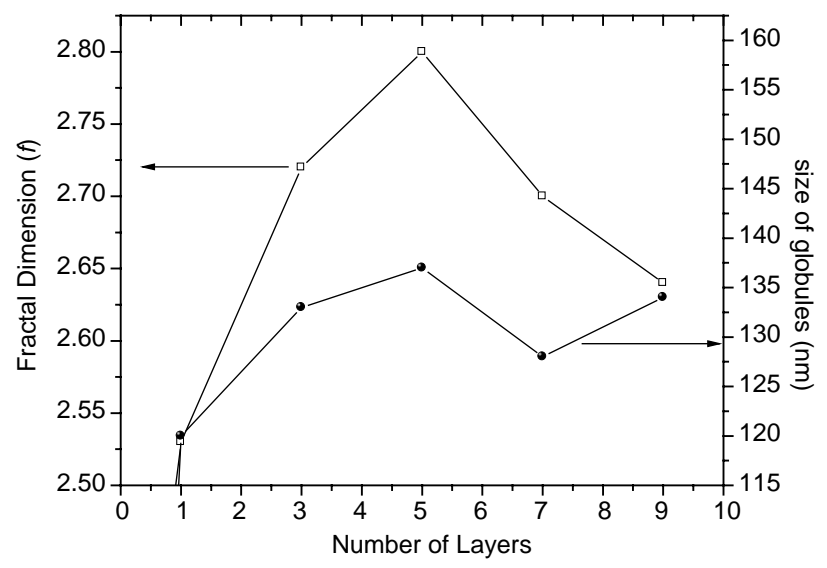

Fig. 10. Fractal dimension and size of the globules vs. number of layers for POEA. layers, the roughness increases, since the flat surface of glass exhibits a very low roughness when compared to POEA layers. However, as the number of POEA layers increases, the voids from a previous layer are filled with the additional POEA and as a consequence the surface roughness tends to decrease.

Fig. 10 shows $f$ increasing with the number of POEA layers deposited, until five layers, and then decreasing. Analogously to the roughness, at the first layers deposited a greater number of irregularities appear. With additional layers, such irregularities are filled with polymeric material and the film surface tends to be flatter, i.e. the fractal dimension tends toward 2. In the $3 \mathrm{D}$ growth, the growth rates of nuclei are essentially equal or comparable in the direction perpendicular to the substrate surface. However, in the 2D growth, the nuclei grow more quickly in the parallel direction than in the perpendicular direction until they meet each other and overlap.

\section{Conclusions}

Layer-by-layer films of poly(o-ethoxyaniline) POEA were studied by AFM and morphological parameters such as grain size, roughness, fractal dimension and relative surface coverage were evaluated. Overall, a globular morphology was observed, typical of a nucleation adsorption process, where the first polymer chains are anchored to the substrate and serve as nuclei for further polymer adsorption. The roughness of the layers increases within the first minutes reaches a maximum value and then remains constant after $20 \mathrm{~min}$ of adsorption. At the beginning, few globules are adsorbed and therefore the roughness is large. As the adsorption continues, the voids between globules are filled with more polymeric material and consequently roughness decreases. We find that the kinetics theory, developed to explain thin film growth, provides a qualitative explanation of the time dependence of island nucleation and growth in the late-growth regime and also the island density in the aggregation regime. A similar behavior is observed for the fractal dimension, which increases for the first minutes of adsorption and then tends towards an average value between 2 and 3. For the multilayer films, the globular morphology appears for all the POEA layers deposited. Film roughness increases up to five layers and then decreases as the number of POEA layers deposited increases. The irregularities, such as voids and islands, are constantly filled with polymeric material and the film surface becomes smoother. The fractal dimension behaves in the same manner, reaching its maximum with five layers and then decreasing to a value between 2 and 3 , typical of a planar surface containing irregularities. The study of polymer adsorption by atomic force microscopy is of key importance to understand the adsorption mechanism and surface properties of thin film produced by layer-by-layer deposition.

\section{Acknowledgements}

Financial assistance from $\mathrm{CNPq}, \mathrm{CNPq} / \mathrm{CT}$-Hidro, IMMP/MCT, FAPESP, Rede Nanobiotec/CNPq/MCT (Brazil) is gratefully acknowledged. 


\section{References}

[1] Oliveira Jr ON, He J-A, Zucolotto V, Balasubramanian S, Li L, Nalwa HS, et al. In: Kumar J, Nalwa HS, editors. Handbook of polyelectrolytes and their applications, 1st ed, vol. 1. Los Angeles, CA: American Scientific Publishers; 2002. p. 1-37.

[2] Roberts GG. Langmuir-Blodgett films. New York: Plenum Press; 1990.

[3] Decher G, Hong JD, Schmitt J. Thin Solid Films 1992;210(1-2):831-5.

[4] Stockton WB, Rubner MF. Macromolecules 1997;30(9):2717-25.

[5] Raposo M, Pontes RS, Mattoso LHC, Oliveira Jr ON. Macromolecules 1997;30(20):6095-101.

[6] Pontes RS, Raposo M, Camilo CS, Dhanabalan A, Ferreira M, Oliveira Jr ON. Phys Status Solidi A 1999;173(1):41-50.

[7] Binnig G, Quate CF, Gerber C. Phys Rev Lett 1986;56(9):930-3.

[8] Cheung JH, Stockton WB, Rubner MF. Macromolecules 1997;30(9): $2712-6$.

[9] de Souza NC, Silva JR, Rodrigues CA, Costa LF, Giacometti JA, Oliveira Jr ON. Thin Solid Films 2003;428(1-2):232-6.

[10] Lvov Y, Decher G. Kristallografiya 1994;39(4):696-716.

[11] Gómez-Rodriguez JM, Baró AM, Vazquez L, Salvarezza RC, Vara JM, Arvia AJ. J Phys Chem 1992;96(1):347-50.

[12] Kobiki Y, Suzuki A. Int J Adhes Adhes 1999;19(5):411-6.
[13] Mattoso LHC, Manohar SK, MacDiarmid AG, Epstein AJ. J Polym Sci, Part A: Polym Chem 1995;33(8):1227-34.

[14] Venancio EL, Paterno LG, Consdin Filho N, Borato CE, Filmino A, Mattoso LHC. J. Braz. Chem. Soc. 2005;16(3B):558-68.

[15] Silk T, Hong Q, Tamm J, Compton RG. Synth Met 1998;93(1): 59-64.

[16] Doudevski I, Schwartz DK. Appl Surf Sci 2001;175:17-26.

[17] Leite FL, Borato CE, Oliveira ON, Hermann PSP, Simões ML, Martin Neto L, Mattoso LHC. Interface phenomena on conducting polymer films investigated with atomic force spectroscopy, in 12th International Symposium on Electrets 2005, pp300.

[18] Amar JG, Family F, Lam PM. Phys Rev B 1994;50(12):8781-97.

[19] Harrison JA, Thirsk HR. Electro analytical chemistry. vol. 5. New York: Marcel Dekker; 1971 p. 67.

[20] Kamal MR, Tang Z, Huang T. Int Polym Proc 2001;16(4):376-87.

[21] Leite FL, Herrmann PSP, Borato CE, Oliveira ON, Mattoso LHC. Application of atomic force microscopy on adsorption process: characterization and modeling. Brazilian MRS Meeting $2004 \mathrm{p}$. 179.

[22] Chesters S, Wang H, Kasper G. Solid State Technol 1991;34(1):73-7.

[23] Bhaushan B. Handbook of micro/nanotribology. New York: CRC Press; 1995.

[24] Paterno LG, Mattoso LHC. Polymer 2001;42(12):5239-45. 